\title{
Parenting Goals: Links with Parenting Strategies in Caregivers of Children with Autism Spectrum Disorder
}

\author{
Elizabeth $\mathrm{O}^{\prime}$ Nions $^{1,2}$ (D) Francesca Happé ${ }^{3}$ - Julie Segers ${ }^{1,4} \cdot$ Essi Viding $^{2} \cdot$ Ilse Noens $^{1,4}$
}

Published online: 26 September 2020

(C) The Author(s) 2020

\begin{abstract}
Objectives Parenting goals describe the outcomes that parents aim for when interacting with their child. They have received little attention in research with caregivers of children with autism spectrum disorder (ASD). The present study explored different types (dimensions) of parenting goals reported by caregivers of children with ASD, investigated links between these parenting goals and child characteristics, and explored whether parenting goals were linked to reported use of particular parenting strategies.

Methods Data from 161 caregivers of children with ASD aged 7-18 years revealed two goal dimensions: "Norm Adherence Goals" (i.e. the perceived importance of the child cooperating with the parent, respecting their authority, and behaving well in public); and "Autonomy Support and Relationship Goals" (i.e. the perceived importance of promoting the child's resilience, wellbeing, and the quality of the parent-child relationship).

Results Parents who reported that norm adherence goals were particularly important to them were more likely to endorse parenting strategies such as setting rules, using rewards, and giving punishments. These parents also reported using less "Accommodation" (e.g. making allowances for their child, being flexible, avoiding triggers). Autonomy support and relationship goals were very strongly endorsed by nearly all parents, and we therefore could not find strong links between the importance of these goals and parenting strategies.

Conclusion These findings suggest that parenting goals might be importantly linked to parenting strategies and could be considered in personalizing interventions in clinical practice. Given that parents' cognitions about their child's behaviour and their role as parents likely interact with and influence their parenting goals, we argue that future research should investigate parenting goals, cognitions, and behaviours in parallel.
\end{abstract}

Keywords Parenting goals $\cdot$ Parenting behaviour $\cdot$ Norm adherence $\cdot$ Autonomy support $\cdot$ Accommodation $\cdot$ Reinforcement . Autism spectrum disorder

Autism spectrum disorders (ASD) are neurodevelopmental disorders characterised by difficulties with communication

Elizabeth O’Nions

e.o'nions@ucl.ac.uk

1 Parenting and Special Education Research Unit, Faculty of Psychology and Educational Sciences, KU Leuven, Leuven, Belgium

2 Developmental Risk \& Resilience Unit, Clinical, Educational, and Health Psychology Research Department, Division of Psychology and Language Sciences, University College London, London, UK

3 Social, Genetic and Developmental Psychiatry Centre, Institute of Psychiatry, Psychology and Neuroscience, King's College London, London, UK

4 KU Leuven, Leuven Autism Research (LAuRes), Leuven, Belgium and socialisation, and rigid and repetitive behaviours (American Psychiatric Association 2013). Behaviour perceived as difficult or challenging by caregivers, including extreme emotional reactivity, is common in children with ASD (e.g. Mazefsky et al. 2018a, b). Recent work has investigated how parents try to manage difficult behaviour and reactivity (O'Nions et al. 2018). Reports suggest that punishment can elicit escalation in difficult behaviour in some children with ASD who have emotion regulation difficulties (e.g. Agazzi et al. 2013; Armstrong et al. 2015; Lucyshyn et al. 2007). This is consistent with accounts suggesting that problem behaviour such as aggression is often motivated by anxiety or distress in children with ASD. Escape from parental demands negatively reinforcing problem behaviour, and punishment can increase anxiety and the motivation to engage in escape-motivated 
problem behaviour (Lucyshyn et al. 2015; O'Nions and Noens 2018).

Studies of parenting in families of children with ASD suggest that many parents adapt to their child by adopting a more flexible approach, such as allowing their child more leeway with rules and expectations, compared with parents of controls who are neurotypical (Maljaars et al. 2014). Boonen et al. (2015) reported less "structuring" by parents during interactions in a sample of 30 mothers of school-aged children with ASD compared with 39 parents of typically developing controls. The authors speculated that a more flexible approach avoided intensifying the child's frustration. There also appear to be positive links between child reactivity and parental "accommodation", a set of parenting strategies that includes adjusting expectations, following the child's rules for how things should be done, and avoiding the child's triggers (O'Nions et al. 2020). Accommodation is reportedly often motivated by the need to prevent or terminate child distress or aggression (Feldman et al. 2019; Lucyshyn et al. 2004; O'Nions et al. 2018). This places a significant burden on families in the long-term, because it requires considerable effort to proactively accommodate the child's specific preferences by reorganizing family activities around the child's requirements (e.g. Lucyshyn et al. 2004).

Researchers are increasingly becoming interested in the thoughts and beliefs (cognitions) that influence parenting behaviour, including "parenting goals". Goals can be thought of as "a means by which emotions, motives, or values are translated into actions" (Bugental and Johnston 2000, p. 318), and parenting goals have been described as "the outcomes that parents hope to achieve while they are interacting with their children" (Hastings and Grusec 1998, p. 465).

Research on goals that influence human social behaviour more generally has suggested various categories, including self-image goals (e.g. achieving status, making oneself acceptable to others), growth-oriented goals (e.g. learning from mistakes), and compassionate goals (e.g. being good to others, avoiding doing harm) (Crocker and Canevello 2008; Dykman 1998). The small numbers of studies that have investigated parenting goals have proposed broadly similar typologies (Dix 1992; Hastings and Grusec 1998). Parenting goals labelled "parent-centred" include promoting the child's obedience and reducing discomfort to parents caused by problem behaviour (Dix 1992; Hastings and Grusec 1998). Parentcentred goals may also include feeling competent in the parenting role, and thus maintaining a positive self-image.

Parenting goals labelled "child-centred" include prioritising the child's emotional needs, supporting the child's wellbeing, and teaching them important values (Dix 1992; Hastings and Grusec 1998). A third set of goals, which include promoting close relationships among family members, has been labelled "relationship-centred" (Grusec and Goodnow 1994; Hastings and Grusec 1998). Child- and relationship- centred goals likely draw on both growth- and compassionoriented motivations.

Parenting goals are believed to be dynamic, with priorities shifting towards the parent's, child's, or family's interests depending on the context, and the specific needs that this triggers in the parent (Coplan et al. 2002; Horvath and Lee 2015, Horvath et al. 2015; Kuczynski 1984; Bugental and Johnston 2000). Research on parenting goals in families of typically developing children has suggested that short-term parent-centred goals (e.g. reducing discomfort caused by the child's problem behaviour) become more salient in public situations (Hastings and Grusec 1998), and might also do so under time constraints, or when parents are experiencing stress.

Parenting goals are likely to be an important influence on parenting behaviour (Bugental and Johnston 2000). Dix (1992) argued that parenting behaviours are selected based on the parenting goals that predominate in a particular context. Monitoring whether the parenting behaviour elicits a childresponse consistent with the goal, stimulates positive or negative affect (Dix 1992); a feedback process that influences subsequent goal selection.

Goals that are most salient to parents might promote particular types of parenting behaviour (Hastings and Grusec 1998). For example, goals that centre on the child's emotional wellbeing, or the quality of the relationship with the child, might motivate non-contingent reinforcement, affection, and warmth, to strengthen affiliative bonds within the family. Growth-seeking goals may motivate parental engagement, reasoning, and autonomy support (Hastings and Grusec 1998). Parent-centred or self-image goals may motivate parenting strategies that seek to reduce difficult behaviour, such as contingent reinforcement to discourage behaviours that provoke annoyance or discomfort.

Taking an individual differences perspective, Hastings and Grusec (1998) investigated how choosing to prioritise parent-, child-, or relationship-centred goals related to behaviours chosen to manage a conflict in vignettes depicting interactions between a parent and a 6-year-old child. Respondents who prioritised parent-centred goals over other goal types were more likely to use power assertion (e.g. giving a command, withdrawing privileges, or using physical force), and less likely to use reasoning or to show acceptance. Prioritising childor relationship-centred goals was linked to selection of more facilitative and responsive strategies (e.g., acknowledging, negotiating, or giving comfort), and to less power assertion (Hastings and Grusec 1998). Encouraging respondents to focus on parent-centred goals led to greater negative affect and less empathy for the child, whilst encouraging a focus on relationship-centred goals had the opposite effect.

Findings suggesting a link between parents' goals and their chosen parenting strategies have also been reported recently by Kirby et al. (2019). In this study of 151 mothers of children 
aged 3-9 years old ( $42 \%$ of whom had concerns about their child's social, emotional, or behavioural development), selfimage goals (i.e. prioritising one's own needs at the expense of others; Crocker et al. 2009) were linked to more reported psychological control as a means of influencing the child's behaviour, and to less facilitative parenting. These effects were present even after accounting for the child's level of difficult behaviour. In contrast, compassionate goals were linked to less parental use of psychological control as a behavioural management strategy, and to more facilitative parenting.

Despite increasing research interest in parenting behaviour in parents of children with ASD, we know very little about parenting goals in this population. The demands of parenting a child with ASD can be very high, particularly when the child shows difficult behaviour (e.g. O'Nions et al. 2018), which has been linked to high levels of parenting stress, and reductions in parents' sense of competence (e.g. Hastings and Brown 2002; Yorke et al. 2018). Despite this, studies suggest that mothers of children with ASD show similar or greater levels of positive parenting compared with those seen in typically developing children (e.g. Boonen et al. 2015; van Esch et al. 2018; Maljaars et al. 2014). It appears that many parents make considerable efforts to overcome the challenges they face to provide an optimal rearing environment for their child.

Despite this, when parents are stretched beyond their limits, some may experience an increase in the salience of parentcentred goals, including the need for feelings of competence and autonomy. Lack of these feelings, for example when the child displays high levels of difficult behaviour, could trigger feelings of shame, which have in turn been linked to more coercive parenting (e.g. Mills et al. 2007). In line with this, findings from a study of 95 parents of children with ASD suggested that parents' unmet needs for feelings of competence, connectedness, and autonomy partially mediated the link between child problem behaviour and controlling parenting (Dieleman et al. 2018). Therefore, one might expect that unmet needs could, in some contexts and for some parents, lead to prioritization of parent-centred goals over other goal types, which in turn may promote more coercive parenting.

To the best of our knowledge, only one previous study has investigated parenting goals specifically in caregivers of children with ASD. Conti (2015) compared compassionate and self-image parenting goals and behaviours in 74 mothers of children with ASD aged 5 to 18 years, and 46 mothers of matched neurotypical children. They (like Kirby et al. 2019), used an adapted version of Crocker and Canevello's (2008) compassionate and self-image goals questionnaire, which included items such as "In the past month, in your role as a parent, how often did you want to or try to have compassion for your child(ren)'s mistakes or weaknesses" (compassionate) or "how often did you want to or try to get your child(ren) to behave in a way that make(s) you proud?" (self-image). Mothers of children with ASD reported more compassionate parenting goals and behaviours than mothers of neurotypical children, although no differences were found for self-image goals. This study reported that compassionate goals positively predicted personal and family life satisfaction and parenting efficacy, whereas self-image goals did not (Conti 2015).

The aim of the present study was to explore the relations between parenting goals and parenting behaviour in parents of children with ASD. Rather than measure parents' intentions regarding their own behaviour or experience, we focused on goals regarding child behaviour. The first aim of this study was to explore the component structure of a new set of questions tapping these goals. The second aim was to explore whether child characteristics (e.g. ASD severity) were related to parenting goals. The third aim was to investigate whether parenting goals were linked to parenting behaviour, and, in particular, the strategies that parents reported using to manage difficult behaviour. The final aim was to explore the extent to which parenting goals were useful in predicting parenting strategies, over and above other (e.g. child) factors. We predicted that child behavioural goals linked to parent-centred concerns would be associated with greater use of rules and reinforcement, and that goals more related to promoting the child's wellbeing would be linked to more accommodation, adaptation, and positive parenting.

\section{Methods}

\section{Participants}

This work was part of a longitudinal study investigating parenting and child behaviour, approved by the KU Leuven Societal and Public Ethics Committee. Informed consent was obtained from all individual participants included in the study. The full sample (393 parents/caregivers) was recruited via electronic links posted on social networks by the research team, and by other organisations for parents of children with ASD and/or other neurodevelopmental profiles, including extreme/"pathological" demand avoidance. Participating parents were encouraged to share information about the study with others to facilitate recruitment.

The present analysis used data from 161 parents/caregivers of children aged 7 to 18 years. Inclusion criteria for this study were that (a) the parent/caregiver was living with the child full-time or part-time; (b) the parent/caregiver reported that the child had a diagnosis of ASD; (c) the child's score on the Social Communication Questionnaire-lifetime version (SCQ; Rutter et al. 2003) was 12 or above (e.g. Mazefsky et al. 2018b). Median age-band for the 161 respondents was 40-44 years at study enrolment (12 months prior to collection of these data). Mean age for the 161 young people in the sample at the time these data were collected was 12 years 
3 months (range 7 years 1 month-17 years 8 months). Further sample characteristics are provided in Table 1.

\section{Procedure}

Data were gathered through self-administered questionnaires collected electronically, completed by the child's parent/

Table 1 Sample characteristics

\begin{tabular}{|c|c|c|}
\hline Child characteristics & $N$ & $\%$ \\
\hline Male & 113 & 70 \\
\hline Female & 46 & 29 \\
\hline Other/ complex gender identity & 2 & 1 \\
\hline \multicolumn{3}{|l|}{ Reported diagnoses } \\
\hline ASD & 161 & 100 \\
\hline Anxiety & 51 & 32 \\
\hline ADHD & 46 & 29 \\
\hline PDA/ demand avoidance & 57 & 35 \\
\hline Mood disorder or major depression & 15 & 9 \\
\hline Conduct problems/ ODD/ challenging behaviour & 11 & 7 \\
\hline Mild intellectual disability & 20 & 12 \\
\hline Moderate intellectual disability & 16 & 10 \\
\hline Severe intellectual disability & 8 & 5 \\
\hline \multicolumn{3}{|l|}{ Reported use of language to communicate ${ }^{1}$} \\
\hline Mostly verbal & 157 & 98 \\
\hline Mostly non-verbal & 4 & 2 \\
\hline \multicolumn{3}{|l|}{ Reported academic level $^{1}$} \\
\hline Similar to /ahead of mainstream peers & 56 & 35 \\
\hline Similar to mainstream peers apart from specific difficulties & 45 & 28 \\
\hline Slightly behind mainstream peers & 11 & 7 \\
\hline Markedly behind peers & 31 & 19 \\
\hline Very far behind mainstream peers & 18 & 11 \\
\hline \multicolumn{3}{|c|}{ Reported independence in daily living skills (e.g. dressing, washing) ${ }^{1}$} \\
\hline Similar to or ahead of others of their age & 30 & 19 \\
\hline Need a bit more help than others their age & 46 & 29 \\
\hline Need a lot more help than others their age & 68 & 42 \\
\hline Completely dependent on parental/carer assistance & 17 & 11 \\
\hline \multicolumn{3}{|l|}{ Child educational setting } \\
\hline Mainstream school & 75 & 47 \\
\hline Specialist or alternative setting & 58 & 36 \\
\hline Home-educated & 22 & 14 \\
\hline Left school or not currently enrolled in school & 6 & 4 \\
\hline \multicolumn{3}{|l|}{ Parent/caregiver characteristics ${ }^{1}$} \\
\hline Female gender & 156 & 97 \\
\hline Residing in the UK & 158 & 98 \\
\hline Child's natural parent & 153 & 95 \\
\hline Married/ cohabiting & 124 & 78 \\
\hline White European ethnicity & 156 & 97 \\
\hline Post school-age qualifications & 117 & 73 \\
\hline
\end{tabular}

${ }^{1}$ These data were collected on entry to the study caregiver. Questionnaires included items on child, parent/ caregiver, and family characteristics. Analyses were conducted using Stata 16.

\section{Measures}

Measures analysed in the present study are described in detail below. Total scores were calculated by taking the mean of items for which responses were provided and multiplied by the total number of items in the scale, provided that at least $50 \%$ of items were present.

Parenting Goals Twelve questions relevant to parenting goals (also applicable to caregivers) were developed by the research team for the purposes of this study. The aim was to generate items that would span a range of parenting goals pertaining to concrete child behaviours of motivational significance to parents of school-aged children. Items generated for the purpose of the present study covered the importance to the parent that the child: (a) adhere to social conventions ( 2 items); (b) cooperate with parents/respect their authority ( 2 items); (c) develop trust in/a positive relationship with the parent ( 2 items); (d) get on with peers (1 item); (e) develop resilience ( 2 items); (f) develop autonomy ( 2 items); and ( $\mathrm{g}$ ) engage in enjoyable activities $(1 \mathrm{item})$. Each item was rated from 0 to 5 : disagree strongly (0), disagree moderately (1), disagree slightly (2), agree slightly (3), agree moderately (4), and agree strongly (5).

Parenting Behaviours Parenting strategies to manage problem behaviour were assessed using the Parenting Strategies Questionnaire (PSQ, O'Nions et al. 2020). Each item was rated on a 5-point scale: never (0), almost never (1), sometimes (2), often (3), and always (4). The PSQ has three subscales: accommodation (15 items, Cronbach's $\alpha=.89$; e.g. "Adjust expectations depending on the child's mood"); reinforcement approaches (10 items, Cronbach's $\alpha=.83$; e.g. "Remove items or privileges as a punishment"); and reducing uncertainty (6 items, Cronbach's $\alpha=.72$; e.g. "Inform the child in advance about changes in routine") (O'Nions et al. 2020).

Other parenting behaviours were measured using the Parenting Behaviour Scale-ASD version (PBS-A, Lambrechts et al. 2011; Maljaars et al. 2014). Responses to items are made on a 5-point scale: never/almost never (1), rarely (2), sometimes (3), often (4), and always (5). To give the scale a minimum score of 0 , we recoded items so that never/almost never received a score of 0 .

Exploratory factor analysis of the Parenting Behaviour Scale-ASD was conducted in a separate dataset consisting of 862 parents of children aged 6 to 16 years (mean $=11.12$ ), of whom 509 had ASD. This was done to reduce the number of items collected for this scale. Based on those results, we 
included eight (of 11) positive parenting items (e.g. "When my child has a problem, we look at different possible solutions together"; Cronbach's $\alpha=.79$ ), six (all) discipline items (e.g. "When my child does something that is not allowed, I give him/her a punishment"; Cronbach's $\alpha=.89$ ), and four (of 6) rules items (e.g. "I make agreements with my child about how he/she should behave"; Cronbach's $\alpha=.76$ ), plus ten (of 11) stimulating development items (e.g. "When someone is crying, I explain to my child what that person is feeling and why"; Cronbach's $\alpha=.88$ ), and six (of 9) adapting the environment items (e.g. "I make sure that my child is not overstimulated"; Cronbach's $\alpha=.60$ ).

Child ASD Severity Child ASD severity was measured using the Social Communication Questionnaire (SCQ) - Lifetime Version (Rutter et al. 2003), a 40-item parent-report screening measure quantifying behaviours associated with ASD. Nineteen yes/no items focus on the entire developmental history and 21 on the child's behaviour when he/she was aged 4 to 5 years. Thirty-nine of the 40 items contribute to the total score, indexing ASD severity (Cronbach's $\alpha=.80$ ).

Child Reactivity The Emotion Dysregulation Inventory (EDI) was used to quantify emotional reactivity (Mazefsky et al. $2018 \mathrm{a}, \mathrm{b})$. Items are rated on a 5-point thermometer scale: not at all (0), mild (1), moderate (2), severe (3), and very severe (4), with severity capturing frequency and intensity. The reactivity subscale ( 24 items) measures high arousal behaviours, such as aggression and emotional outbursts. The dysphoria subscale (6 items) measures lower arousal behaviours. Only data on reactivity were analysed in the present study (Cronbach's $\alpha=.98$ ).

Background Characteristics Data on family characteristics, including number of children in the family, parent/caregiver (and cohabiting partner's) age, educational qualifications, and employment, were also collected. A proxy indicator of socio-economic status (SES) was generated by combining information on educational qualifications and employment, using the Office for National Statistics coding tool (https:// onsdigital.github.io/dp-classification-tools/standardoccupational-classification/ONS_NSSEC_discovery_tool. html).

\section{Data Analyses}

Principal components analysis was used to explore the dimensionality of parenting goal items. We then used a paired samples $t$ test to compare endorsement of the two goal types. Next, we used $t$ tests and correlation analyses to explore links between parenting goals and both child characteristics and parenting strategies. Finally, we used regression analyses to examine whether parenting goals could predict reported use of particular parenting strategies over and above child characteristics.

\section{Results}

\section{Descriptive Characteristics of the Sample}

The 161 children with ASD in this study sample reportedly showed high levels of reactivity, with 52 (32\%) showing reactivity at least one standard deviation above published ASD norms for children aged 4-20 years (Mazefsky et al. 2018a). In addition, 148 (92\%) were reported to exhibit difficult or challenging behaviour.

\section{Examining Endorsement of Parenting Goal Items}

Mean endorsement rates indicated that parents in this sample agreed that all of the parenting goals were important (see Table 2). Item 10 ("It is important to me that my child gets on well academically") was least endorsed: rated as "Strongly Disagree" by 16 parents (10\%), and "Strongly Agree" by only eight parents $(5 \%)$.

\section{Dimensionality of Parenting Goal Items}

Principal components analysis (PCA) with Varimax rotation was used to explore covariance among parenting goal-items. Inspection of the scree plot (Cattell 1966) informed extraction of the optimal number of subscales. The scree plot showed an elbow at the third eigenvalue, so PCA was repeated with the number of extracted components constrained to two, collectively explaining $43 \%$ of the measured variance.

PCA informed the inclusion of items in relevant subscales. Applying cutoffs used by Benson (2010), items were retained in a subscale if they loaded $\geq .|40|$ onto one of the components and $<.|40|$ onto the other component. Eleven of the 12 items met these criteria (see Table 3 ).

The first subscale ( 5 items) included items covering the perceived importance to the parent/caregiver that the child be cooperative, respect their authority, and behave well in public. Although resembling "parent-centred goals", we labelled the scale "Norm Adherence Goals". This was because, given the exploratory nature of the study and the fact that we did not adopt a systematic item generation strategy, we cannot assume that we have captured the full range of goals described elsewhere, or potentially construed as "parent-centred". Goals included in this subscale also appear to reflect child-centred motivations, since the desire for the child to cooperate and behave well in public may be due to the need to promote the child's socialization, rather than primarily to reinforce parents' self-image (Hastings and Grusec 1998). Therefore, it seems most appropriate to describe 
Table 2 Mean endorsement rate for parenting goals items

\begin{tabular}{lllll}
\hline Item & Mean score & SD & Median agreement level & Score range \\
\hline ("It is important to me that my child...") & & & & \\
(1) Respects my authority & 3.35 & 1.37 & Moderately \\
(2) Trusts me & 4.94 & 0.24 & Strongly & $0-5$ \\
(3) Learns to work things out for him/herself & 4.39 & 0.75 & Strongly & $4-5$ \\
(4) Behaves well in public or in front of friends and family & 3.36 & 1.20 & Moderately \\
(5) Has the freedom to choose what he/she will/ will not do & 3.96 & 0.84 & Moderately \\
(6) Cooperates with me (e.g. does not cause a delay) & 2.99 & 1.15 & Slightly \\
(7) Can cope in stressful situations & 4.37 & 0.86 & Strongly \\
(8) Enjoys spending time with me & 4.42 & 0.78 & Strongly & $0-5$ \\
(9) Can bounce back from setbacks & 4.57 & 0.59 & Strongly & $1-5$ \\
(10) Does well academically & 2.61 & 1.39 & Slightly \\
(11) Does things that he/she enjoys on a regular basis & 4.71 & 0.53 & Strongly \\
(12) Gets on well with peers & 3.84 & 1.02 & Moderately & $0-5$ \\
\hline
\end{tabular}

these goals in terms of the nature of desired outcomes rather than inferences regarding underlying motivations.

The second subscale (6 items) included items covering the perceived importance of prioritising child resilience and wellbeing, and the quality of the relationship with the child. Although resembling previous accounts of "child-/relationshipcentred" goals, this scale was termed "Autonomy Support and Relationship Goals", because we have likely not covered the full range of goals previously described as child-/relationshipcentred. As noted above, we are also unable to infer the nature of the underlying motivations related to these goals. For example, the goal of promoting the child's resilience and increasing their wellbeing could be both "child-centred" and "parent-centred", since child emotion dysregulation may produce considerable parental anxiety and problems in day-to-day life. Therefore, we chose a label that focuses on desired outcomes.

We first compared endorsement for the two parenting goal subscales. Mean subscale scores were 16.16 for norm adherence goals $(\mathrm{SD}=4.36$, range $=1-25$, mean item score $=3.23$, item $\mathrm{SD}=.87$ ), and 27.39 for autonomy support and relationship goals $(\mathrm{SD}=2.39$, range $=21-30$, mean item score $=4.57$, item $\mathrm{SD}=.40)$, the latter suggesting a ceiling effect. Autonomy support and relationship goals were significantly more endorsed than norm adherence goals $(t(160)=20.05$, $p<.001$, Cohen's $d=1.98$ ), suggesting that, on the whole, parents/caregivers value their child's autonomy and the

Table 3 Component loadings and item-total correlations for parenting goal subscales

\begin{tabular}{|c|c|c|c|}
\hline Item ("It is important to me that my child....") & Component 1 & Component 2 & Item-total correlation for subscale \\
\hline \multicolumn{4}{|l|}{ Subscale 1: Norm adherence goals (Cronbach's alpha $=0.75$ ) } \\
\hline (6) Cooperates with me (e.g. does not cause a delay). & 0.78 & 0.02 & 0.73 \\
\hline (4) Behaves well in public or in front of friends and family. & 0.75 & 0.07 & 0.74 \\
\hline (1) Respects my authority. & 0.75 & 0.12 & 0.75 \\
\hline (10) Does well academically. & 0.62 & 0.07 & 0.71 \\
\hline (12) Gets on well with peers. & 0.48 & 0.36 & 0.59 \\
\hline \multicolumn{4}{|c|}{ Subscale 2: Autonomy support and relationship goals (Cronbach's alpha $=0.65)$} \\
\hline (9) Can bounce back from setbacks. & 0.20 & 0.71 & 0.73 \\
\hline (2) Trusts me. & -0.09 & 0.60 & 0.45 \\
\hline (8) Enjoys spending time with me. & 0.07 & 0.59 & 0.62 \\
\hline (3) Learns to work things out for him/herself. & 0.27 & 0.59 & 0.68 \\
\hline (7) Can cope in stressful situations. & 0.25 & 0.57 & 0.70 \\
\hline (11) Does things that he/she enjoys on a regular basis. & -0.20 & 0.56 & 0.48 \\
\hline (5) Has the freedom to choose what he/she will/ will not do. & -0.33 & 0.35 & - \\
\hline
\end{tabular}

Component loadings $\geq .|40|$ are presented in italics. Item 5 (highlighted in bold) loaded significantly onto neither component 
quality of the relationship with their child more than they value their child's adherence to norms. However, the two goal types were modestly positively related $(r=.30, p<.001)$.

\section{Relations Between Parenting Goals and Child Characteristics}

Next, we explored links between parenting goals and child characteristics. Since this analysis was exploratory, we note all nominally significant results below. The Bonferroni adjusted $p$ value was $p<.003$ (16 comparisons).

Norm adherence goals and autonomy support and relationship goals were both unrelated to child age $\left(r_{\mathrm{s}}<|.05|, p \mathrm{~s}>.3\right)$ and did not differ between caregivers of male and female children ( $p>.3$ ). Norm adherence goals did not differ between caregivers of children with and without intellectual disability (ID) $(p>.3)$. Autonomy support and relationship goals were slightly lower for parents of children with an ID diagnosis $(26.84, \mathrm{SD}=2.52)$ compared with those without, but although this effect was moderate, it did not reach significance (27.59, $\mathrm{SD}=2.32 ; t(159)=1.80, p=.07$, Cohen's $d=.36$ ).

Norm adherence goals were significantly more endorsed by parents of children at mainstream school $(17.57, \mathrm{SD}=$ $3.50)$, compared with those not at mainstream $(14.93, \mathrm{SD}=$ 4.67), $t(159)=4.02, p<.001$, Cohen's $d=.63$; significant at the Bonferroni-adjusted threshold). Norm adherence goals were also less endorsed by parents of children with lower estimated academic skills $\left(r_{\mathrm{s}}=-.20, p<.05\right)$, and less independence in daily living skills $\left(r_{\mathrm{s}}=-.21, p<.01\right)$. Autonomy support and relationship goals were unrelated to these variables $\left(r_{\mathrm{s}}<|.08|, p \mathrm{~s}>.3\right)$. Neither goal type was linked to ASD severity or reactivity $(r<|.08|, p \mathrm{~s}>.3)$.

\section{Relations Between Parenting Goals and Parenting Behaviours}

Next, we explored the link between parenting goals and parenting behaviours and strategies, including those used to manage behaviour experienced as difficult or challenging. Norm adherence goals were positively related to reported parental behaviour involving reinforcement approaches, discipline, and rules (all $r \mathrm{~s}>.40$, Table 4, Fig. 1). In addition, norm adherence goals were modestly negatively related to reported use of accommodation; $(r=-0.27)$.

No other correlations survived correction for multiple comparisons, although norm adherence goals were positively linked to two subscales: stimulating development and adapting the environment at a nominal threshold. Autonomy support and relationship goals showed weak positive links with four subscales: reinforcement approaches, rules, positive parenting, and stimulating development at a nominal threshold.
Given the skew in several variables, analyses were repeated using Spearman's correlations. For relations between each parenting goal-type and parenting behaviour dimension, all but one Spearman coefficient was within 0.04 of the Pearson's estimate. For the link between norm adherence goals and reducing uncertainty, the Spearman's estimate was smaller by $.06\left(r_{\mathrm{s}}=0.07\right)$.

\section{Predicting Parenting Behaviours from Parenting Goals}

Finally, we used hierarchical regression to examine whether norm adherence goals and autonomy support and relationship goals predicted reported use of parenting strategies, beyond effects of child ASD symptoms, reactivity, reported academic level and independence in daily living tasks. The impact of child-level variables on parenting is discussed in depth elsewhere (O'Nions et al. 2020), so the focus here is on additional variance captured when parenting goals were added to the models.

Since demographic factors (e.g. parent age, educational level, SES, family size) were unrelated to parenting strategies in previous analyses (O'Nions et al. 2020), we did not include parent/family level covariates in regression models. The Bonferroni adjusted $p$ value for a significant change in $R^{2}$ was $p<.003$ (16 models of interest). Within models, the adjusted significance threshold for individual predictors was $p<.006$ ( 8 predictors). Only results significant at Bonferroni-adjusted thresholds are described in the text.

We first considered the Parenting Strategies Questionnaire subscales, which focus particularly on approaches to manage behaviour experienced as difficult or challenging (Table 5). Norm adherence goals negatively predicted accommodation (explaining an extra $5 \%$ of the variance); and positively predicted reinforcement approaches (explaining an extra $25 \%$ of the variance). Norm adherence goals did not predict reducing uncertainty. Contrary to our hypotheses, autonomy support and relationship goals did not predict accommodation or reducing uncertainty and did not meet the Bonferroni-adjusted significance threshold as a predictor for reinforcement approaches.

Next, we considered the Parenting Behaviour ScaleASD version subscales, which focus on both general and more ASD adapted parenting dimensions. Norm adherence goals positively predicted discipline and rules, explaining an extra $22 \%$ and $16 \%$ of the variance respectively. Norm adherence goals did not significantly predict positive parenting, stimulating development or adapting the environment. Autonomy support and relationship goals did not significantly predict scores on any PBS subscales at Bonferroni-adjusted thresholds (see Table 6). 
Table 4 Pearson correlation coefficients between parenting goals and parenting behaviours

$\begin{array}{llllllllll}1 & 2 & 3 & 4 & 5 & 6 & 7 & 8 & 9 & 10\end{array}$

Parenting Goals

1. Norm adherence goals 1

2. Autonomy support goals $\quad 0.30 * * * \quad 1$

Parenting Strategies Questionnaire

$\begin{array}{llllll}\text { 3. Accommodation } & -0.27 * * * & -0.06 & 1 & & \\ \text { 4. Reinforcement approaches } & 0.46^{* * *} & 0.20^{*} & -0.17^{*} & 1 & \\ \text { 5. Reducing uncertainty } & -0.13 & -0.01 & 0.63^{* * *} & 0.04 & 1\end{array}$

Parenting Behaviour Scale-ASD version

\begin{tabular}{|c|c|c|c|c|c|c|c|c|c|c|}
\hline 6. Discipline & $0.49 * * *$ & $0.19 *$ & $-0.28 * * *$ & $0.73 * * *$ & -0.10 & 1 & & & & \\
\hline 7. Rules & $0.42 * * *$ & $0.21 * *$ & -0.07 & $0.65 * * *$ & 0.14 & $0.48 * * *$ & 1 & & & \\
\hline 8. Positive parenting & 0.10 & $0.16^{*}$ & 0.04 & $0.45 * * *$ & $0.27 * * *$ & $0.21 * *$ & $0.60 * * *$ & 1 & & \\
\hline 9. Stimulating development & $0.18^{*}$ & $0.20 *$ & $0.18 *$ & $0.45 * * *$ & $0.38 * * *$ & $0.25 * *$ & $0.64 * * *$ & $0.75 * * *$ & 1 & \\
\hline 10. Adapting the environment & $0.16^{*}$ & 0.02 & $0.16^{*}$ & $0.50 * * *$ & $0.36^{* * *}$ & $0.24 * *$ & $0.56^{* * *}$ & $0.45 * * *$ & $0.55 * * *$ & 1 \\
\hline
\end{tabular}

$* * * p<0.001, * * p<0.01, * p<0.05$. Bonferroni adjusted significance is $p<0.001$ (significant associations in italics)

\section{Discussion}

Parenting goals in parents of children with ASD have received very little research attention, despite their potential importance for understanding parenting behaviour. The first aim of this study was to explore the dimensional structure of items measuring parenting goals pertaining to achievement of specific child outcomes (e.g. adherence to norms, cooperation, trust in parents, resilience, autonomy) in parents of children with ASD.
Our parenting goal items formed two subscales: "norm adherence goals" and "autonomy support and relationship goals". Items in the norm adherence goals subscale measured the degree to which parents/caregivers considered it important that the child cooperate with them, respect their authority, and behave well in public. These goals broadly resembled previous accounts of "parent-centred" or "self-image" parenting goals, although they may also reflect "child-centred" socialization goals (Hastings and Grusec 1998).
Fig. 1 Norm adherence goals score plotted against discipline score
Norm Adherence Goals Score Plotted Against Discipline Score

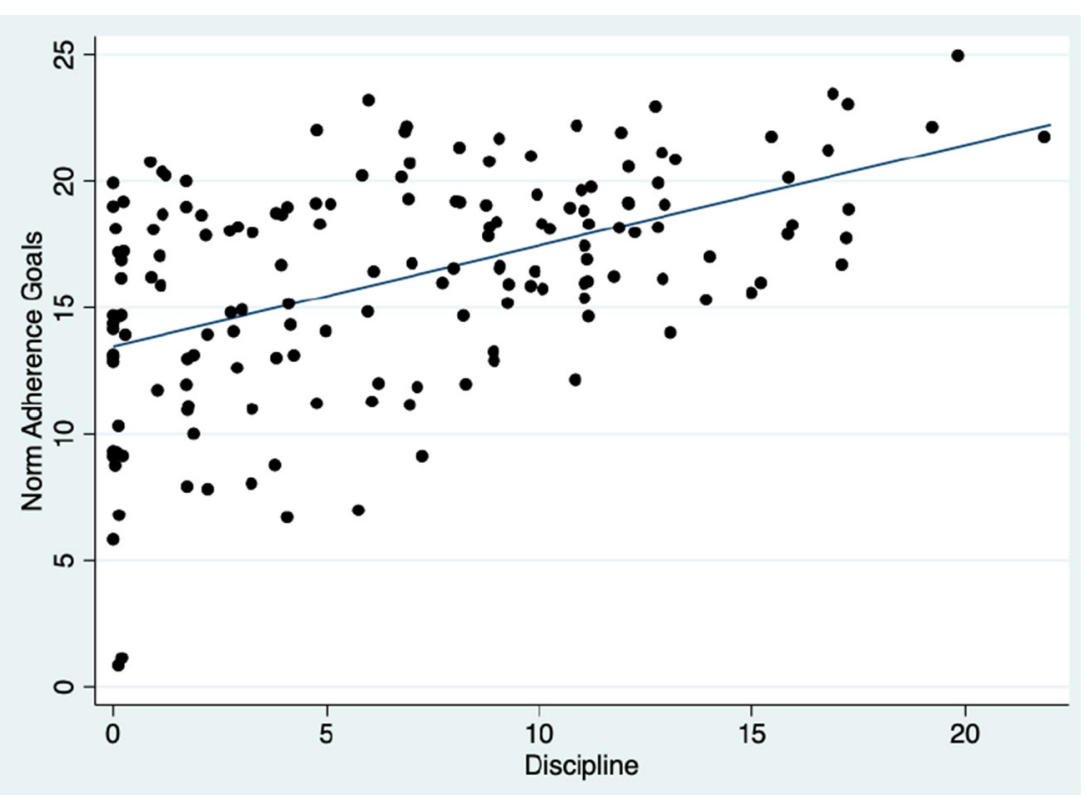

Note $r=.49, \mathrm{p}<.001$ 
Table 5 Models predicting PSQ subscales from child factors and parenting goals

\section{Child variables only}

Child variables + norm adherence goals

(1) Predicting accommodation

Child age
Gender
Lower academic level
Lack of independence
ASD severity
Reactivity
Norm adherence goals
Autonomy support goals
$R^{2}$

\section{$-0.04$}

0.04

0.02

$0.22 * *$

$-0.15$

$0.50 * * *$

$-$

$-$

$0.36^{* * *}$

(2) Predicting reinforcement approaches

$\begin{array}{ll}\text { Child age } & -0.16^{*} \\ \text { Gender } & -0.18 \\ \text { Lower academic level } & 0.13 \\ \text { Lack of independence } & -0.07 \\ \text { ASD severity } & 0.18^{*} \\ \text { Reactivity } & 0.05\end{array}$

$\begin{array}{ll}\text { Norm adherence goals } & - \\ \text { Autonomy support goals } & - \\ R^{2} & 0.10^{*}\end{array}$

(3) Predicting reducing uncertainty

Child age
Gender
Lower academic level
Lack of independence
ASD severity
Reactivity
Norm adherence goals
Autonomy support goals
$R^{2}$

0.04
0.09
-0.06
0.10
0.14
$0.27 * *$
-
-
$0.12 * *$

$\begin{array}{ll}-0.04 & -0.04 \\ -0.02 & 0.04 \\ -0.02 & 0.02 \\ 0.17 * & 0.22 * * \\ -0.14 & -0.15 \\ 0.51 * * * & 0.50 * * * \\ -0.24 * * * & - \\ - & -0.03 \\ 0.41 * * * \dagger & 0.36 * * *\end{array}$

$-0.17 *$

$-0.15$

$-0.16$

0.11

$-0.05$

$0.18 *$

0.06

$-$

$0.19 *$

$0.35 * * *^{\dagger} \quad 0.13 * *$

$0.04 \quad 0.04$

$0.06 \quad 0.09$

$\begin{array}{ll}-0.07 & -0.06\end{array}$

$0.08 \quad 0.10$

$0.15 \quad 0.14$

$0.27 * * \quad 0.27 * *$

$-0.11$

$\begin{array}{ll}- & 0.01\end{array}$

$0.13 * * \quad 0.12 * *$

$†$ Change in $R^{2}$ significant at $p<0.003 . * * * p<0.001, * * p<0.01, * p<0.05$. All variables standardised apart from gender

Autonomy support and relationship goals tapped the degree to which parents/caregivers considered it important to promote their child's resilience and wellbeing, and develop the quality of their relationship with the child; which broadly resembles previous accounts of "child-" and "relationshipcentred" goals, or "compassionate"/"growth-oriented" goals. We chose descriptive labels for these subscales given the ambiguity regarding underlying motivations, and our lack of certainty regarding whether these item sets comprehensively reflect goal-types previously identified under these labels.

Consistent with work by Conti (2015), these findings suggest that parenting goals found in parents of children with ASD overlap with those seen in broader samples of parents (which may, of course, have included small numbers of parents of children with ASD, e.g. Hastings and Grusec 1998; Kirby et al. 2019). In the present sample, parents varied in their endorsement of norm adherence goals. However, autonomy support and relationship goals were almost unanimously endorsed, resulting in a ceiling effect. This result is similar to the finding reported by Conti (2015), which indicated that parents of children with ASD showed higher endorsement of compassionate vs. self-image parenting goals (Cohen's $d=$ 1.67).

\section{Parenting Goals and Parenting Behaviour}

A key question of interest in this study was whether parenting goals can predict parenting behaviour. We found that norm adherence goals positively predicted reported use of reinforcement approaches, rules, and discipline. Variation in norm adherence goals explained $16-25 \%$ of the variance in these parenting strategies over and above child factors. 
Table 6 Models predicting PBS-A subscales from child factors and parenting goals

Child variables only Child variables + norm adherence goals Child variables + autonomy support goals

\begin{tabular}{|c|c|c|c|}
\hline \multicolumn{4}{|l|}{ (1) Predicting discipline } \\
\hline Child age & 0.06 & 0.05 & 0.07 \\
\hline Gender & -0.27 & -0.14 & -0.26 \\
\hline Lower academic level & 0.07 & 0.14 & 0.05 \\
\hline Lack of independence & $-0.26^{* *}$ & $-0.16^{*}$ & $-0.25 * *$ \\
\hline ASD severity & 0.06 & 0.04 & 0.07 \\
\hline Reactivity & 0.09 & 0.07 & 0.10 \\
\hline Norm adherence goals & - & $0.49^{* * *}$ & - \\
\hline Autonomy support goals & - & - & $0.17 *$ \\
\hline$R^{2}$ & 0.07 (n0.s0.) & $0.29 * * * \dagger$ & $0.10^{*}$ \\
\hline \multicolumn{4}{|l|}{ (2) Predicting rules } \\
\hline Child age & -0.01 & -0.01 & -0.00 \\
\hline Gender & -0.28 & -0.17 & -0.26 \\
\hline Lower academic level & -0.06 & 0.00 & -0.08 \\
\hline Lack of independence & -0.12 & -0.03 & -0.10 \\
\hline ASD severity & 0.16 & 0.14 & 0.16 \\
\hline Reactivity & 0.08 & 0.07 & 0.09 \\
\hline Norm adherence goals & - & $0.42 * * *$ & - \\
\hline Autonomy support goals & - & - & $0.21 * *$ \\
\hline$R^{2}$ & $0.05(\mathrm{n} 0 . \mathrm{s} 0)$. & $0.21 * * * \dagger$ & $0.09^{*}$ \\
\hline \multicolumn{4}{|c|}{ (3) Predicting positive parenting } \\
\hline Child age & -0.03 & -0.03 & -0.02 \\
\hline Gender & -0.22 & -0.20 & -0.20 \\
\hline Lower academic level & 0.01 & 0.02 & 0.00 \\
\hline Lack of independence & -0.11 & -0.09 & -0.10 \\
\hline ASD severity & 0.16 & 0.16 & 0.17 \\
\hline Reactivity & 0.07 & 0.07 & 0.08 \\
\hline Norm adherence goals & - & 0.09 & - \\
\hline Autonomy support goals & - & - & 0.15 \\
\hline$R^{2}$ & $0.04(\mathrm{n} 0 . \mathrm{s} 0)$. & $0.05(\mathrm{n} 0 . \mathrm{s} 0)$. & $0.07(\mathrm{n} 0 . \mathrm{s} 0)$. \\
\hline \multicolumn{4}{|c|}{ (4) Predicting stimulating development } \\
\hline Child age & -0.05 & -0.05 & -0.04 \\
\hline Gender & -0.13 & -0.08 & -0.11 \\
\hline Lower academic level & -0.08 & -0.05 & -0.09 \\
\hline Lack of independence & -0.06 & -0.03 & -0.05 \\
\hline ASD severity & 0.12 & 0.11 & 0.12 \\
\hline Reactivity & $0.17 *$ & 0.16 & $0.18^{*}$ \\
\hline Norm adherence goals & - & $0.17 *$ & - \\
\hline Autonomy support goals & - & - & $0.21 * *$ \\
\hline$R^{2}$ & $0.05(\mathrm{n} 0 . \mathrm{s} 0)$. & 0.08 (n0.s0.) & $0.09^{*}$ \\
\hline \multicolumn{4}{|c|}{ (5) Predicting adapting the environment } \\
\hline Child age & $-0.20 * *$ & $-0.21 * *$ & $-0.20 *$ \\
\hline Gender & 0.04 & 0.09 & 0.04 \\
\hline Lower academic level & 0.02 & 0.05 & 0.02 \\
\hline Lack of independence & -0.07 & -0.04 & -0.07 \\
\hline ASD severity & $0.32 * * *$ & $0.31 * * *$ & $0.32 * * *$ \\
\hline Reactivity & 0.11 & 0.11 & 0.11 \\
\hline Norm adherence goals & - & $0.19 *$ & - \\
\hline Autonomy support goals & - & - & 0.02 \\
\hline$R^{2}$ & $0.15^{* * * *}$ & $0.18^{* * * *}$ & $0.15^{* * *}$ \\
\hline
\end{tabular}

$\dagger$ Change in $R^{2}$ significant at $p<0.003 . * * * p<0.001, * * p<0.01, * p<0.05$. All variables standardised apart from gender

One possible explanation for these findings is that parents who prioritise adherence to norms use strategies like setting boundaries, discipline, and contingent reward to motivate the child to comply or engage in desirable behaviour. Another possibility is that parents who value norm adherence make greater demands of their child with ASD that trigger problem behaviour, requiring more frequent use of behavioural control strategies. A third possibility is that parents of children with
ASD who value adherence to norms view certain management strategies, such as time-out, as "punishment", rather than seeing it more as "thinking time", and thus endorse certain question items differently. Qualitative, observational, and longitudinal research designs could be helpful in teasing apart these possibilities.

As well as positively predicting reinforcement-based strategies, norm adherence goals were a modest negative predictor 
of accommodation. Accommodation describes seeking to prevent problematic episodes by being flexible, making allowances for the child, and avoiding encounters with the child's triggers. Thus, this finding is broadly in line with results from Hastings and Grusec (1998), which suggested that goals surrounding child obedience (like norm adherence goals) were negatively related to accepting or understanding responses to child problem behaviour (like accommodation).

Contrary to our hypotheses, autonomy support and relationship goals did not significantly predict individual variation in parenting strategies. This was likely due to ceiling effects, meaning that we were unable to capture sufficient variation using this scale. Future studies using alternative means to capture variation, such as asking parents to rank or prioritise goals in particular situations (e.g. as per Hastings and Grusec 1998), or providing a more comprehensive assessment of relevant goals using systematically derived assessment tools, may be necessary to measure this dimension sensitively.

The present findings suggest that parents' motivations may be a key factor in determining their child management approaches, which has potential implications for clinical practice. In particular, reinforcement-based approaches, which are the mainstay of many parenting interventions, may lack appeal for families for whom norm adherence is not a key priority. Therefore, it may be helpful for interventionists to take into account parenting goals to personalize interventions, and thus offer the best fit for families. Indeed, previous work suggests that interventions can be strengthened by including adaptations to accommodate parenting goals and family-specific perspectives (e.g. McCabe et al. 2005; McCabe and Yeh 2009; McCabe et al. 2012).

\section{Parenting Goals and Child Factors}

In terms of links between parenting goals and child factors, norm adherence goals were $20 \%$ higher in parents of children at mainstream school compared with those not currently educated in mainstream, or not in receipt of education. Norm adherence goals were also endorsed less by parents of children reported to have lower ability/less independence in daily living skills, although these results did not survive Bonferroni correction. These findings tentatively suggest that parents might adapt their goals related to their estimation of their child's capabilities. Parents of children in mainstream schools may also recognise that following rules is necessary for their child's inclusion. Alternatively, parents who value norm adherence less may seek educational provision outside of mainstream.

Notably, parenting goals did not differ in relation to the severity of the child's ASD or their level of reactivity. This suggests that parenting goals are (at least in part) a product of parents' own personalities, beliefs, and value systems, interacting with parents' experiences and their own and their children's environment. The consequence is that parents of children with ASD likely manage similar child behaviours in very different ways. A further possibility is that, for many families, parenting goals no longer play a dominant role in how parents respond when the child displays very high levels of reactivity. Convergent evidence suggests that reductions in child reactivity act as a powerful negative reinforcer for parents, such that various forms of accommodation (e.g. withdrawal of demands, provision of preferred activities) become a routine that overshadows cognitions that typically inform their behaviour with their child (Dumas 2005). Notably, Dumas (2005) highlight that, once established, such routines are usually impervious to change using rewards and punishments. They recommend an alternative set of strategies, specifically, facilitative listening, distancing, and motivated action plans to establish more positive routines (Dumas 2005). Future research should therefore consider how parents' own value systems, their views regarding drivers of child problem behaviour, and the habitual patterns that may have established outside of their conscious awareness, influence both their parenting goals and parenting behaviour.

\section{Limitations and Future Directions}

A key limitation of this study is that, since it was exploratory, we did not take a systematic approach to identify relevant parenting goal items. Conducting a qualitative survey and synthesis of goals pertaining to specific child outcomes would be of considerable value in building capacity to systematically uncover parenting goal dimensions pertaining to child outcomes.

A second limitation is that we did not include a control group of parents of matched children who are neurotypical, or compare parenting goals for siblings in the same family, where one is neurotypical and one has ASD. Since some parents of children with ASD anecdotally report that using reinforcement based (and particularly punitive) approaches to attempt to discourage difficult behaviour would lead to intolerable levels of stress (Lucyshyn et al. 2004), we speculate that some parents of children with ASD may choose to place less emphasis on norm adherence goals compared with what they might for a sibling who is neurotypical. Comparative studies are needed to test this possibility.

A further limitation is that we relied on parental self-report rather than implicit or observational measures of parenting behaviours. Therefore, associations may have been inflated due to common-rater bias. However, since we detected differential links between norm adherence goals and specific parenting strategies, common-rater bias cannot account for these results, as one might expect it to inflate associations across all dimensions.

Our data cannot provide a complete picture of parenting in relation to child behaviour, since only one parent provided 
information about their parenting goals. Since parents may adapt their parenting to offset the style of their partner (e.g. by being more accommodative to compensate for an authoritarian spouse), future studies should examine parenting and parenting goals in both parents to improve our understanding of family systems (Smetana et al. 2006).

A further consideration is that, in the present study, participants were a community-based sample recruited from online sources (e.g., parent support groups, social network groups). Many participating parents had a particular interest in extreme/"pathological" demand avoidance (PDA), a profile purportedly linked to anxietydriven avoidance of routine demands in ASD, which has stimulated considerable interest and debate in the UK (see e.g. Green et al. 2018; O'Nions and Noens 2018). The PDA-concept involves a formulation of avoidance behaviour that emphasizes child anxiety as the driving factor and, therefore, likely impacts parental cognitions regarding what is at the root of their child's difficulties. Specifically, parents familiar with the PDAconcept may be less likely to attribute child reactivity or avoidance to "willful defiance". Since recommendations for PDA described by caregivers and some clinicians include adopting a child-centred flexible approach (e.g. Christie et al. 2012), we speculate that the present sample may well show lower norm adherence goals compared with epidemiological samples exhibiting similar levels of child reactivity.

Lastly, clinical data (e.g., on child diagnoses, IQ, verbal ability) were not available for this sample. We cannot be sure that gold-standard diagnostic tools were used to inform diagnostic decision-making. Therefore, further studies in clinically well-characterised autism populations recruited using epidemiological sampling are needed to explore possible links between parenting goals, parenting strategies, and child characteristics.

Parenting goals remain under-explored in relation to ASD, despite their relevance to understanding parenting behaviour. Future studies could investigate the intersection between parenting strategies; comparing participants high on both norm adherence and autonomy support and relationship goal types, on each one exclusively, and on neither goal type. This may afford more predictive power than exploring links with dimensions independently.

Parenting goals are likely to be partly influenced by cognitions regarding the child's emotions and the reasons for their behaviour (e.g. see Bugental and Johnston 2000). Several study participants reported anecdotally that fully recognizing and appreciating their child's difficulties had led to a change in their approach to parenting. Some parents mentioned placing less importance and value on others' expectations, judgements, and advice and instead concentrating on developing trust and improving their relationship with their child. Multi-method longitudinal studies are needed to explore whether and how parenting goals evolve in parallel with cognitions and beliefs and impact parenting behaviour. Such studies should also take into account the role of automatic parenting behaviours that reduce or prevent child reactivity, which operate outside of conscious awareness, and therefore may show a mismatch with stated goals (Dumas 2005; Lucyshyn et al. 2015).

Our recently published meta-synthesis (O'Nions et al. 2018) suggested that the demands of parenting children with very high levels of emotion dysregulation can lead parents to focus exclusively on avoiding demands and routines that may provoke child problem behaviour and submitting to their child when he or she engages in problem behaviour to access a want or need. Reports suggest that, in the absence of professional support, this is often perceived as the only conceivable option to prevent family breakdown (e.g. Lucyshyn et al. 2004). However, the sense of entrapment that would result likely has deleterious consequences for long-term parental mental and physical health.

More research is needed to see whether interventions designed to foster certain "parent-centred" parenting goals, such as cultivating detachment from the child's emotional states, or prioritising self-care in parenting contexts, may be helpful in addressing this issue. However, consistent with work suggesting that resilience is heavily impacted by the community and support structures around an individual (Ungar and Theron 2020), these types of "parentcentred" goals may only come online when parents are able to access the emotional and practical support that they require to facilitate more positive interactions with their child and reduce their stress.

Acknowledgements E O'Nions was supported by a postdoctoral fellowship from the M.M. Delacroix Support Fund. We are extremely grateful to the parents/caregivers who took part in this research for their time and efforts. We thank Jane O'Nions and the anonymous reviewers for their very helpful comments on the manuscript.

Authors' Contributions EO contributed to the design of the study, conducted data analyses, and contributed to the writing of the study and the preparation/editing of the manuscript. FH contributed to the design and writing of the study and interpretation of results. JS conducted literature searches and contributed to the writing of the study. EV contributed to the writing of the study and interpretation of results, IN contributed to the design and writing of the study and interpretation of results.

\section{Compliance with Ethical Standards}

Ethics Statement This study was approved by the KU Leuven Societal and Public Ethics Committee.

All procedures performed in studies involving human participants were in accordance with the ethical standards of the KU Leuven Societal and Public Ethics Committee (G-2016-12-712) and with the 1964 Helsinki declaration and its later amendments. 
Informed Consent Statement All participants provided informed consent prior to their participation in the study.

Conflict of Interest The authors declare that they have no conflict of interest.

Open Access This article is licensed under a Creative Commons Attribution 4.0 International License, which permits use, sharing, adaptation, distribution and reproduction in any medium or format, as long as you give appropriate credit to the original author(s) and the source, provide a link to the Creative Commons licence, and indicate if changes were made. The images or other third party material in this article are included in the article's Creative Commons licence, unless indicated otherwise in a credit line to the material. If material is not included in the article's Creative Commons licence and your intended use is not permitted by statutory regulation or exceeds the permitted use, you will need to obtain permission directly from the copyright holder. To view a copy of this licence, visit http://creativecommons.org/licenses/by/4.0/.

\section{References}

Agazzi, H., Tan, R., \& Tan, S. Y. (2013). A case study of parent-child interaction therapy for the treatment of autism spectrum disorder. Clinical Case Studies, 12(6), 428-442. https://doi.org/10.1177/ 1534650113500067.

American Psychiatric Association. (2013). Diagnostic and statistical manual of mental disorders: DSM-5 (5th ed.). Washington, D.C: American Psychiatric Association.

Armstrong, K., DeLoatche, K. J., Preece, K. K., \& Agazzi, H. (2015). Combining parent-child interaction therapy and visual supports for the treatment of challenging behavior in a child with autism and intellectual disabilities and comorbid epilepsy. Clinical Case Studies, 14(1), 3-14. https://doi.org/10.1177/1534650114531451.

Benson, P. R. (2010). Coping, distress, and well-being in mothers of children with autism. Research in Autism Spectrum Disorders, 4(2), 217-228. https://doi.org/10.1016/j.rasd.2009.09.008.

Boonen, H., van Esch, L., Lambrechts, G., Maljaars, J., Zink, I., Van Leeuwen, K., \& Noens, I. (2015). Mothers' parenting behaviors in families of school-aged children with autism spectrum disorder: an observational and questionnaire study. Journal of Autism and Developmental Disorders, 45(11), 3580-3593. https://doi.org/10. 1007/s10803-015-2506-6.

Bugental, D. B., \& Johnston, C. (2000). Parental and child cognitions in the context of the family. Annual Review of Psychology, 51(1), 315344 .

Cattell, R. B. (1966). The scree test for the number of factors. Multivariate Behavioral Research, 1(2), 245-276. https://doi.org/ 10.1207/s15327906mbr0102 10.

Christie, P., Duncan, M., Healy, Z., \& Fidler, R. (2012). Understanding pathological demand avoidance syndrome in children. Jessica Kingsley Publishers.

Conti, R. (2015). Compassionate parenting as a key to satisfaction, efficacy and meaning among mothers of children with autism. Journal of Autism and Developmental Disorders, 45(7), 2008-2018. https:// doi.org/10.1007/s10803-015-2360-6.

Coplan, R. J., Hastings, P. D., Lagacé-Séguin, D. G., \& Moulton, C. E. (2002). Authoritative and authoritarian mothers' parenting goals, attributions, and emotions across different childrearing contexts. Parenting, 2(1), 1-26. https://doi.org/10.1207/ S15327922PAR0201_1.

Crocker, J., \& Canevello, A. (2008). Creating and undermining social support in communal relationships: the role of compassionate and self-image goals. Journal of Personality and Social Psychology, 95(3), 555-575. https://doi.org/10.1037/0022-3514.95.3.555.

Crocker, J., Olivier, M.-A., \& Nuer, N. (2009). Self-image goals and compassionate goals: costs and benefits. Self and Identity, $8(2-3)$, 251-269. https://doi.org/10.1080/15298860802505160.

Dieleman, L. M., De Pauw, S. S. W., Soenens, B., Mabbe, E., Campbell, R., \& Prinzie, P. (2018). Relations between problem behaviours, perceived symptom severity and parenting in adolescents and emerging adults with ASD: the mediating role of parental psychological need frustration. Research in Developmental Disabilities, 73, 21-30. https://doi.org/10.1016/j.ridd.2017.12.012.

Dix, T. H. (1992). Parenting on behalf of the child: empathic goals in the regulation of responsive parenting. In I. V. Sigel, A. V. McGillicuddy-DeLisi, \& T. J. Goodnow (Eds.), Parental belief systems: The psychological consequences for children (pp. 319-346). Erlbaum.

Dumas, J. E. (2005). Mindfulness-based parent training: strategies to lessen the grip of automaticity in families with disruptive children. Journal of Clinical Child and Adolescent Psychology, 34(4), 779791. https://doi.org/10.1207/s15374424jccp3404 20.

Dykman, B. M. (1998). Integrating cognitive and motivational factors in depression: initial tests of a goal-orientation approach. Journal of Personality and Social Psychology, 74(1), 139-158. https://doi.org/ 10.1037//0022-3514.74.1.139.

Feldman, I., Koller, J., Lebowitz, E. R., Shulman, C., Ben Itzchak, E., \& Zachor, D. A. (2019). Family accommodation in autism spectrum disorder. Journal of Autism and Developmental Disorders, 49(9), 3602-3610. https://doi.org/10.1007/s10803-019-04078-x.

Green, J., Absoud, M., Grahame, V., Malik, O., Simonoff, E., Le Couteur, A., \& Baird, G. (2018). Pathological demand avoidance: symptoms but not a syndrome. The Lancet Child \& Adolescent Health, 2(6), 455-464. https://doi.org/10.1016/S2352-4642(18) 30044-0.

Grusec, J. E., \& Goodnow, J. J. (1994). Impact of parental discipline methods on the child's internalization of values: a reconceptualization of current points of view. Developmental Psychology, 30(1), 4 19. https://doi.org/10.1037/0012-1649.30.1.4.

Hastings, R. P., \& Brown, T. (2002). Behavioural knowledge, causal beliefs and self-efficacy as predictors of special educators' emotional reactions to challenging behaviours. Journal of Intellectual Disability Research, 46(Pt 2), 144-150. https://doi.org/10.1046/j. 1365-2788.2002.00378.x.

Hastings, P. D., \& Grusec, J. E. (1998). Parenting goals as organizers of responses to parent-child disagreement. Developmental Psychology, 34(3), 465-479. https://doi.org/10.1037/0012-1649.34.3.465.

Horvath, C. A., \& Lee, C. M. (2015). Parenting responses and parenting goals of mothers and fathers of adolescents. Marriage \& Family Review, 51(4), 337-355. https://doi.org/10.1080/01494929.2014. 955938.

Horvath, C. A., Lee, C. M., \& Bax, K. (2015). How similar are mothers and fathers of young children in their parenting responses and goals? Journal of Child and Family Studies, 24(12), 3542-3551. https:// doi.org/10.1007/s10826-015-0155-1.

Kirby, J. N., Grzazek, O., \& Gilbert, P. (2019). The role of compassionate and self-image goals in predicting psychological controlling and facilitative parenting styles. Frontiers in Psychology, 10. https:// doi.org/10.3389/fpsyg.2019.01041.

Kuczynski, L. (1984). Socialization goals and mother-child interaction: strategies for long-term and short-term compliance. Developmental Psychology, 20(6), 1061-1073. https://doi.org/10.1037/0012-1649. 20.6.1061.

Lambrechts, G., Van Leeuwen, K., Boonen, H., Maes, B., \& Noens, I. (2011). Parenting behaviour among parents of children with autism spectrum disorder. Research in Autism Spectrum Disorders, 5(3), 1143-1152. https://doi.org/10.1016/j.rasd.2010.12.011. 
Lucyshyn, J. M., Irvin, L. K., Blumberg, E. R., Laverty, R., Horner, R. H., \& Sprague, J. R. (2004). Validating the construct of coercion in family routines: expanding the unit of analysis in behavioral assessment with families of children with developmental disabilities. Research and Practice for Persons with Severe Disabilities, 29(2), 104-121. https://doi.org/10.2511/rpsd.29.2.104.

Lucyshyn, J. M., Albin, R. W., Horner, R. H., Mann, J. C., Mann, J. A., \& Wadsworth, G. (2007). Family implementation of positive behavior support for a child with autism. Journal of Positive Behavior Interventions, 9(3), 131-150. https://doi.org/10.1177/ 10983007070090030201.

Lucyshyn, J. M., Fossett, B., Bakeman, R., Cheremshynski, C., Miller, L., Lohrmann, S., et al. (2015). Transforming parent-child interaction in family routines: longitudinal analysis with families of children with developmental disabilities. Journal of Child and Family Studies, 24(12), 3526-3541. https://doi.org/10.1007/s10826-0150154-2.

Maljaars, J., Boonen, H., Lambrechts, G., Van Leeuwen, K., \& Noens, I. (2014). Maternal parenting behaviour and child behaviour problems in families of children and adolescents with autism spectrum disorder. Journal of Autism and Developmental Disorders, 44(3), 501512. https://doi.org/10.1007/s10803-013-1894-8.

Mazefsky, C. A., Day, T. N., Siegel, M., White, S. W., Yu, L., \& Pilkonis, P. A. (2018a). Development of the emotion dysregulation inventory: a PROMIS®ing method for creating sensitive and unbiased questionnaires for autism spectrum disorder. Journal of Autism and Developmental Disorders, 48(11), 3736-3746. https://doi.org/10. 1007/s10803-016-2907-1.

Mazefsky, C. A., Yu, L., White, S. W., Siegel, M., \& Pilkonis, P. A. (2018b). The emotion dysregulation inventory: psychometric properties and item response theory calibration in an autism spectrum disorder sample. Autism Research, 11(6), 928-941. https://doi.org/ 10.1002/aur.1947.

McCabe, K., \& Yeh, M. (2009). Parent-child interaction therapy for Mexican Americans: a randomized clinical trial. Journal of Clinical Child \& Adolescent Psychology, 38(5), 753-759. https:// doi.org/10.1080/15374410903103544.

McCabe, K. M., Yeh, M., Garland, A. F., Lau, A. S., \& Chavez, G. (2005). The GANA program: a tailoring approach to adapting parent child interaction therapy for Mexican Americans. Education and Treatment of Children, 28(2), 111-129.

McCabe, K., Yeh, M., Lau, A., \& Argote, C. B. (2012). Parent-child interaction therapy for Mexican Americans: results of a pilot randomized clinical trial at follow-up. Behavior Therapy, 43(3), 606-618. https://doi.org/10.1016/j.beth.2011.11.001.

Mills, R. S. L., Freeman, W. S., Clara, I. P., Elgar, F. J., Walling, B. R., \& Mak, L. (2007). Parent proneness to shame and the use of psychological control. Journal of Child and Family Studies, 16(3), 359374. https://doi.org/10.1007/s10826-006-9091-4.

O’Nions, E., \& Noens, I. (2018). Commentary: conceptualising demand avoidance in an ASD context - a response to Osman Malik \& Gillian Baird (2018). Child and Adolescent Mental Health, 23(4), 389-390. https://doi.org/10.1111/camh.12287.

O’Nions, E., Happé, F., Evers, K., Boonen, H., \& Noens, I. (2018). How do parents manage irritability, challenging behaviour, noncompliance and anxiety in children with autism spectrum disorders? A meta-synthesis. Journal of Autism and Developmental Disorders, 48(4), 1272-1286. https://doi.org/10.1007/s10803-017-3361-4.

O’Nions, E., Ceulemans, E., Happé, F., Benson, P., Evers, K., \& Noens, I. (2020). Parenting strategies used by parents of children with ASD: differential links with child problem behaviour. Journal of Autism and Developmental Disorders, 50(2), 386-401. https://doi.org/10. 1007/s10803-019-04219-2.

Rutter, M., Bailey, A., \& Lord, C. (2003). The social communication questionnaire. Western Psychological Services.

Smetana, J. G., Campione-Barr, N., \& Metzger, A. (2006). Adolescent development in interpersonal and societal contexts. Annual Review of Psychology, 57, 255-284. https://doi.org/10.1146/annurev.psych. 57.102904.190124.

Ungar, M., \& Theron, L. (2020). Resilience and mental health: how multisystemic processes contribute to positive outcomes. The Lancet Psychiatry, 7, 441-448. https://doi.org/10.1016/S22150366(19)30434-1.

van Esch, L., Vanmarcke, S., Ceulemans, E., Van Leeuwen, K., \& Noens, I. (2018). Parenting adolescents with ASD: a multimethod study. Autism Research, 11(7), 1000-1010. https://doi.org/10.1002/aur. 1956.

Yorke, I., White, P., Weston, A., Rafla, M., Charman, T., \& Simonoff, E. (2018). The association between emotional and behavioral problems in children with autism spectrum disorder and psychological distress in their parents: a systematic review and meta-analysis. Journal of Autism and Developmental Disorders, 48(10), 3393-3415. https:// doi.org/10.1007/s10803-018-3605-y.

Publisher's Note Springer Nature remains neutral with regard to jurisdictional claims in published maps and institutional affiliations. 